\title{
Infected nasopalatine cyst or neoplasm
}

\author{
Anvar Amonov', Bakhrom Yusupov', Akbar Khasanov', Akhmad Madaminov', \\ Zohir Shukurov', Rahimjon Bekmirzaev', Gulrukh Botiralieva', Azizbek Omonov ${ }^{2}$ \\ ${ }^{1}$ Republican Specialized Scientific and Practical Medical Center of Oncology and Radiology, \\ Tashkent, Uzbekistan \\ ${ }^{2}$ Tashkent State Dental Institute, Tashkent, Uzbekistan
}

\section{ABSTRACT}

The cyst is accumulated fluid or mass in a cavity lined by pathological epithelium. The mandible and the maxilla are bones with high prevalence of cyst formation in the human body. Cystic affections, with or without bone destruction of the hard palate, are considered benign tumours. We present the case of a 20-year-old young man with a cystic lesion of the hard palate having lasted for 7 months.

KEYWORDS: maxilla, mandible, cyst, infected cyst.

\section{INTRODUCTION}

Cystic involvement of the maxilla is a benign formation with odontogenic or non-odontogenic origin. The classification of cystic lesions was published in 1992 by the World Health Organization and updated in $2005^{1}$. Odontogenic cysts are: lateral periodontal cyst, gingival cyst of the adult, keratocyst, follicular cyst, cyst rash, infant alveolar cyst, calcified odontogenic cyst, glandular odontogenic cyst, characterised as developmental. Inflammatory cysts are: radicular cysts, residual cysts and paradental cysts. The nasopalatine cyst, the nasopalatine canal cyst, the nasolabial cyst and globulomaxillary cysts are non-odontogenic developmental cysts.

Extracorporeal and intracorporeal factors can provide infection activity and complications of cysts. Suppuration and capsule necrosis can lead to bone destruction: destruction of the hard palate, the floor of the maxillary sinus, the inferior wall of the nasal fossa ${ }^{2,3}$.

The case report presents a male patient with an infected cyst of the maxilla and hard palate. Massive formation in the oral cavity was the main symptom and the reason to present to the Head and Neck Cancer Department.

\section{CASE REPORT}

A 20-year-old male presented with edema of the right side of the face (Figure 1A) and swelling tumour over the hard palate having lasted for 7 months, associated with pain during alimentation. Oropharyngoscopy showed a massive, spherical, $3 \times 4 \mathrm{~cm}$ in size and non-erosive tumour at the level of the hard palate (Figure 1B). Palpation caused pain, and it revealed a tumour of firm consistency and a slight fluctuation in the midline of it. The face skin and the oral mucosa were unchanged, normal in colour, the maxillary incisors with no carious lesions and trauma. The rhinoscopy revealed a deformation of the floor of the right side. The rest of the oral and dental examination was normal.

The CT scan of the head and neck showed a tumour form mass with firm density shadow on the right side, extended to the maxillary sinus, the nasal fossa and the hard palate (Figure 2). On the sagittal slices, a clear bone destruction of the hard palate could be seen. After these investigations, we decided for a diagnostic puncture which was positive. We know from experience that sometimes the content of the tumoral mass can consist in pus or disruption process, which was confirmed.

Preoperative blood investigations (complete hemoleucogram, coagulation tests, biochemical blood tests, etc.) were in normal figures, which confirmed a non-fever clinical form of neoplasm. After the evaluation of the investigation results, the patient was prepared for surgery. Considering a neoplasm process and for less bleeding during surgery, the first step was the ligation of the right external carotid branches.

Corresponding author: Anvar Amonov, MD, Republican Specialized Scientific and Practical Medical Center of Oncology and Radiology of the Ministry of Health of the Republic of Uzbekistan, Shaykhantaur district, 383 Farobi Street, Tashkent, Republic of Uzbekistan 

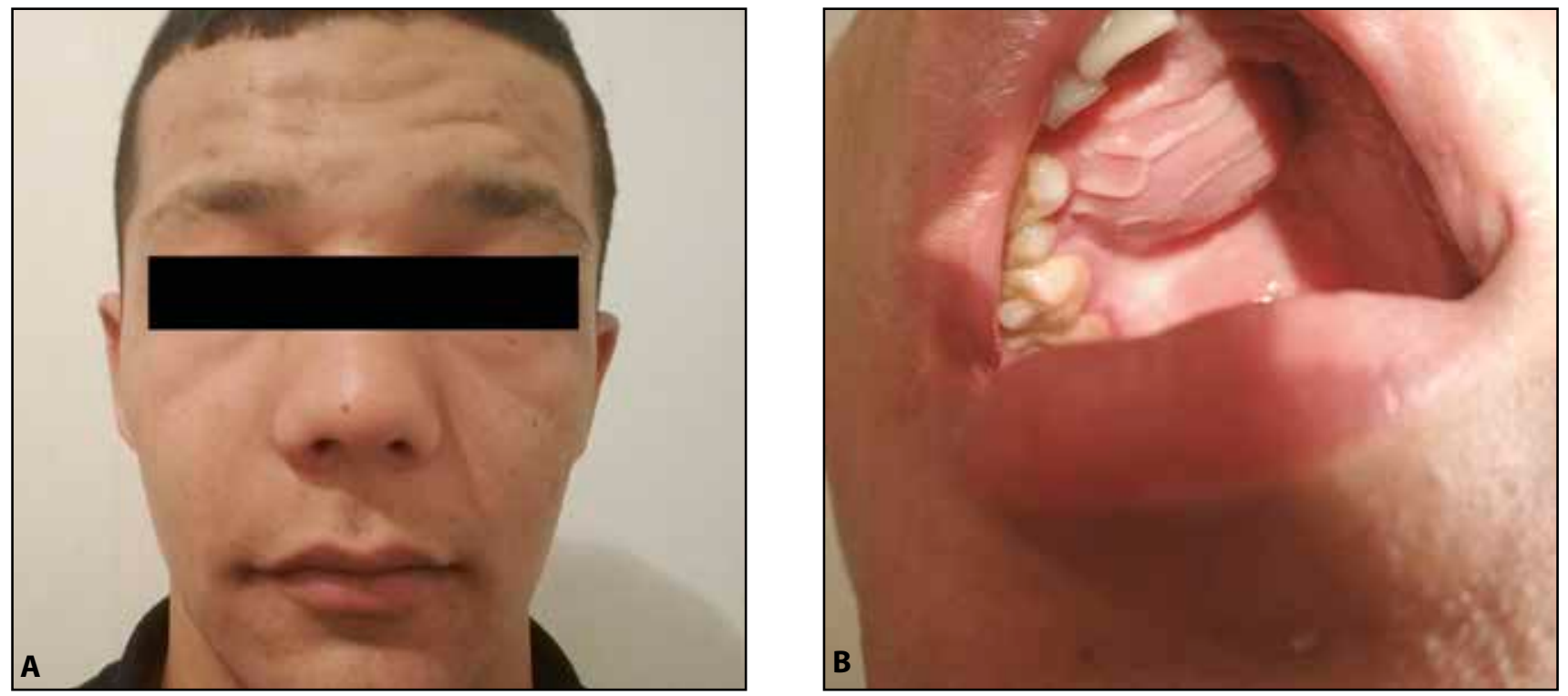

Figure 1 A. Edema of the right side of the face. B. Tumour at the level of the hard palate.
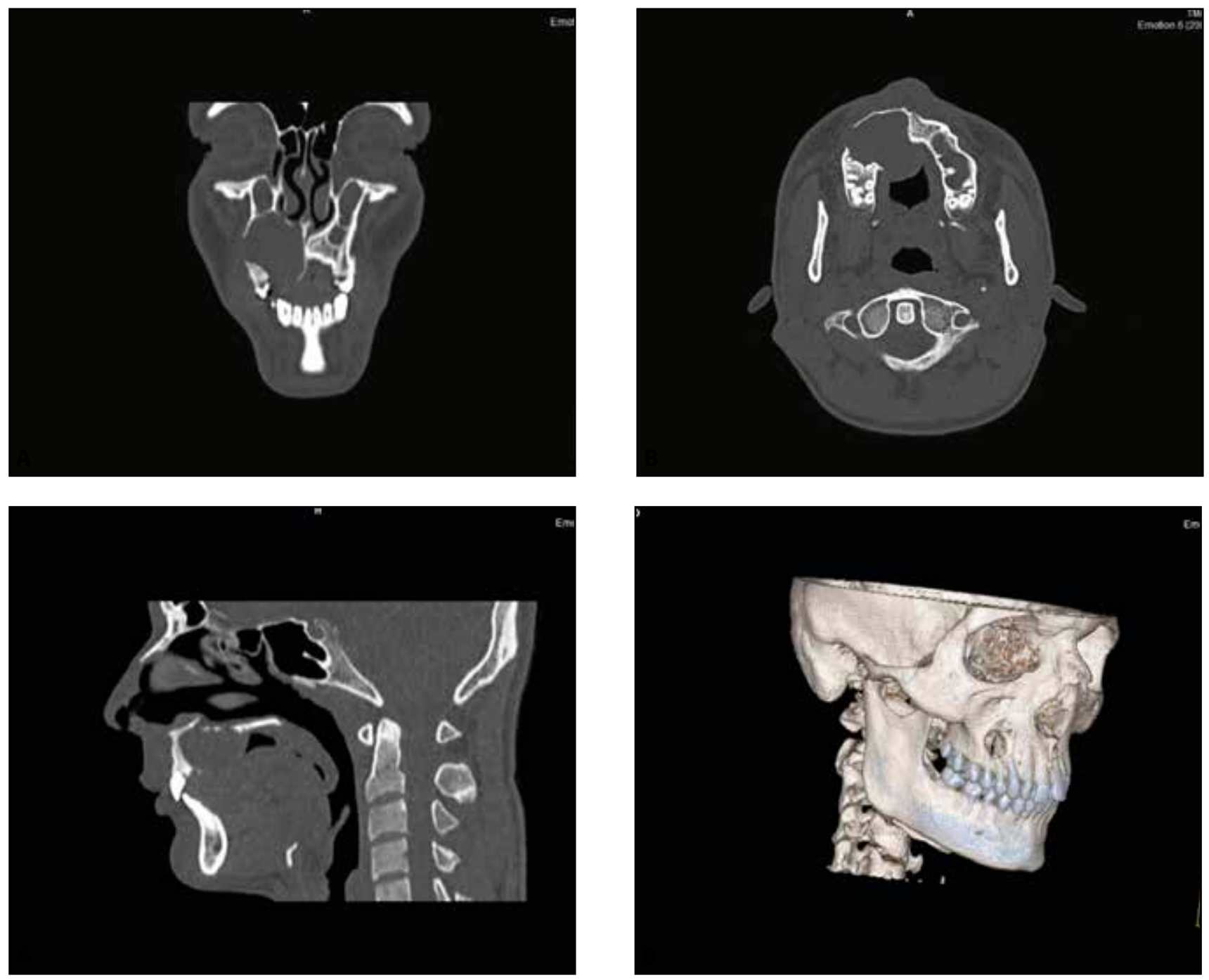

Figure 2 Tumour form mass with firm density shadow on the right side, extended to the maxillary sinus, the nasal fossa and the hard palate. 

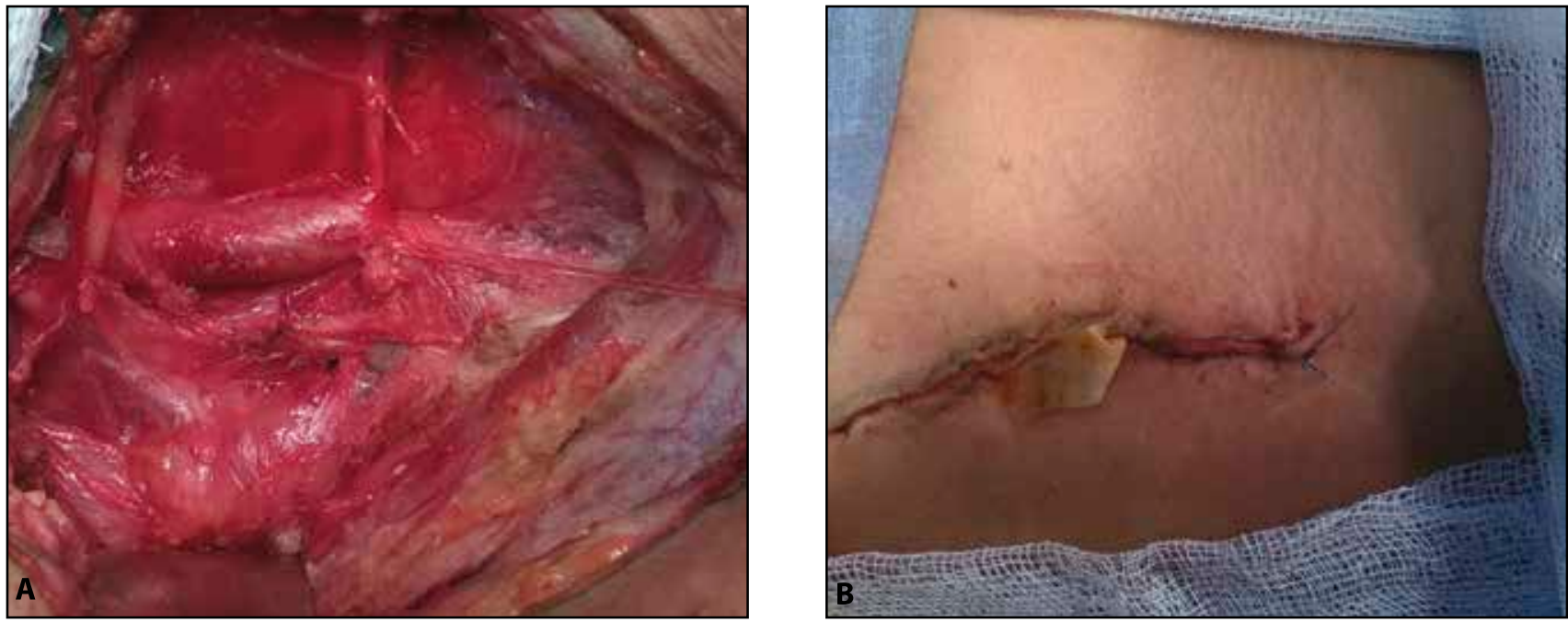

Figure 3 A,B Intraoperative view - Identification of the external carotid artery and the legation of the external carotid artery and its branches. The wound was stitched up.

Surgery. The patient was placed in supine position on the operating table. The head was extended and slight rotated to the left and he was orally intubated. The incision area was marked. An incision of approximately $7-10 \mathrm{~cm}$ started from the retromandibular angle and continued with the anterior margin of the sternocleidomastoid muscle and finished at the level of the cricoid cartilage. The skin, the subcutaneous cellular tissue and the platysma were incised. After the elevation of the skin flaps, the sternocleidomastoid muscle was dissected and lateralized together with the jugular vein. The external carotid artery was identified clearly. The external carotid artery and its branches were legated. Drainage was leaved. The wound was stitched up (Figure 3A,B).

The second part of the surgery was started with an incision from the lateral incisors to the second molar tooth (Figure 4A). The mucosa and the periosteum were ele-

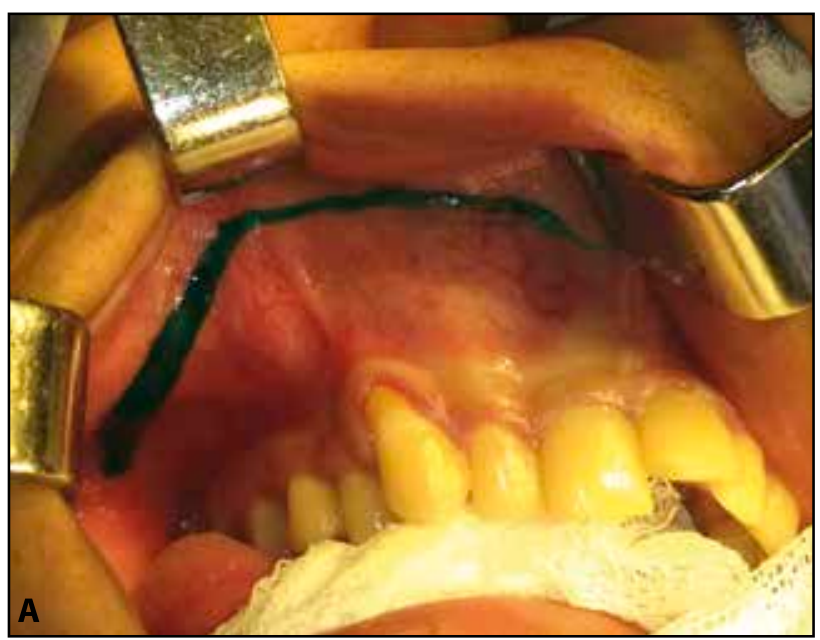

vated and dissected to expose the anterior wall where the destructed area of the right maxillary sinus was. In the canine fossa, the anterior wall of the maxillary sinus was opened. The opening was enlarged (Figure 4B). Pus and hyperplasic internal mucosa were eliminated from the sinus. After elevation and curettage, the palpation of the inferior wall of the maxillary sinus with a ball-point probe showed the totally destructed bone without perforation of the hard palate mucosa. On the medial wall of the sinus (the lateral wall of the nasal fossa), an orifice for drainage to the inferior meatus was created. An antibiotic unguent imbibed pack was leaved inside for homeostasis. The wound was stitched up.

The tumoral mass resected during surgery was send to the histopathological examination where microscope slides were prepared and checked. Under the microscope, the cyst wall was lined by a stratified columnar epithelium,

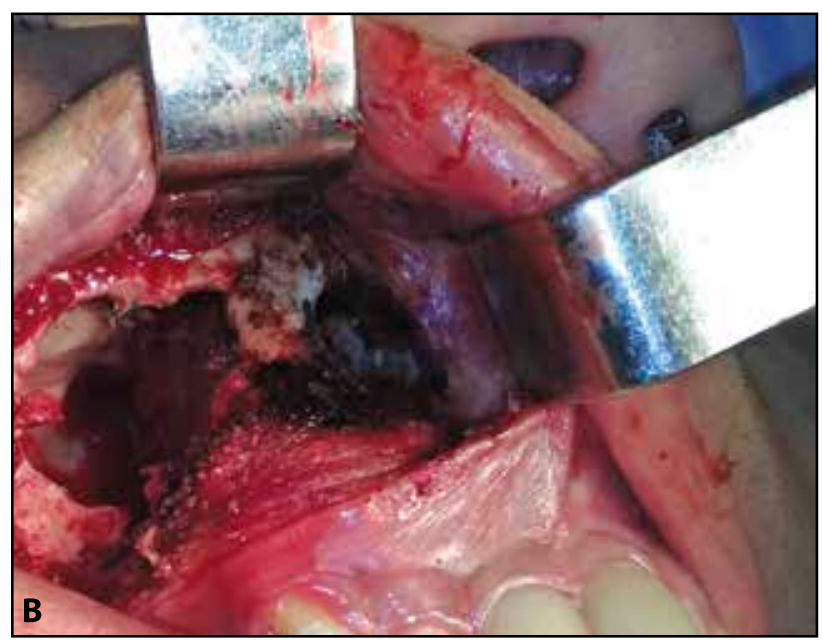

Figure 4 A. Intraoperative view - marked incision area; B. Intraoperative view - enlarged opening of the right maxillary sinus. 

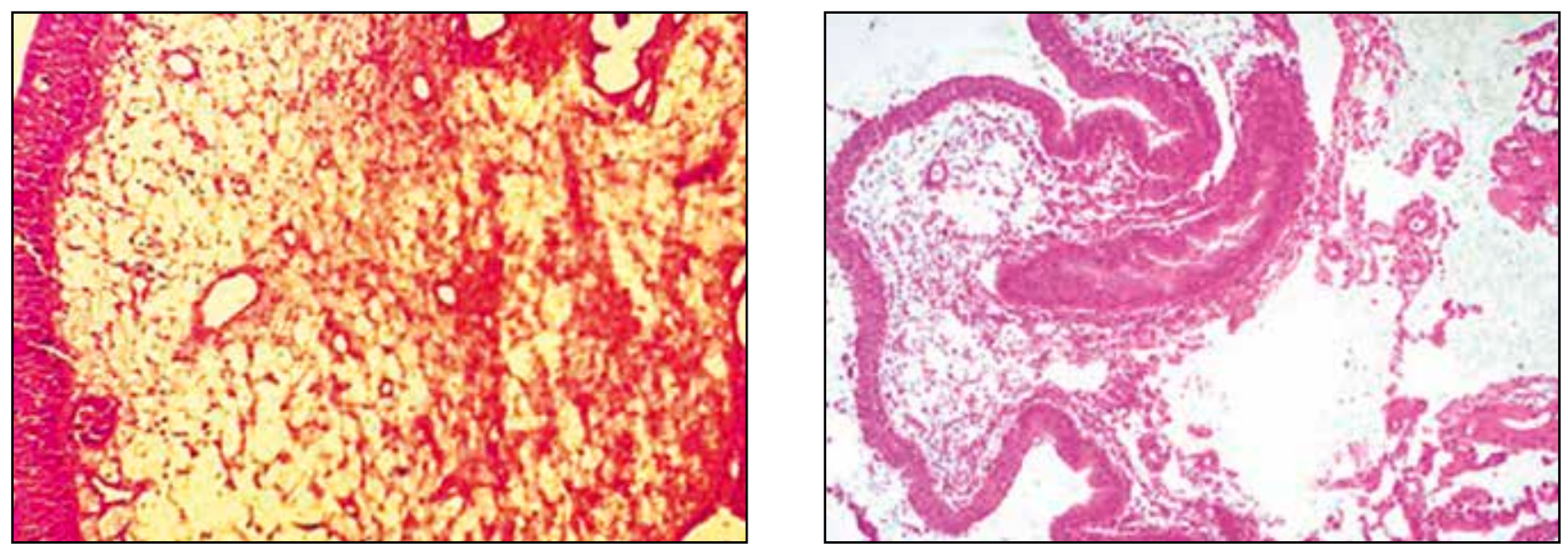

Figure 5 Cyst wall lined by stratified columnar epithelium, under the epithelium fibrous tissue with arteries, veins and glands.

under the epithelium fibrous tissue with arteries, veins and glands, which confirmed a cystic formation (Figure 5).

For one week after surgery the patient was protected with antibiotic and nonsteroidal anti-inflammatory drugs (NSAIDs).

6 months after surgery, the patient showed normal epithelisation of the maxillary sinus and the incision area.

\section{DISCUSSIONS}

Palatine cysts are rare and very uncommon. Most of them are asymptomatic. The etiology of this lesion is still uncertain. Spontaneous cystic degeneration of remnants of the nasopalatine duct and some genetic determinants has been suggested ${ }^{4}$. In this case, the bacterial infection preexisting in the patient could be hypothecated as a possible trigger of the lesion. The purulent process or pus consistency starts osteolysis. The extension of the cyst may penetrate the labial plate and produce a swelling below the maxillary labial frenulum or to one side. Pressure from the cyst on the adjacent palatine nerves that occupy the same canal may cause a burning sensation or numbness over the palatal mucosa. In some cases, such as that of the nasopalatine canal cyst, the cystic fluid may drain into the oral cavity through a sinus tract or a remnant of the nasopalatine duct. The patient usually detects the fluid and reports a salty taste. However, in this case, the process location was not in the midline and it had no pathological orifice.

A well-shaped compact formation with firm consistency and non fever evolution made us to take into consideration a tumoral process. Benign tumours of the maxillary sinus are very rare, most of them are osteomas. Epithelioma is a common name for different epithelial tumours and it is the most common neoplasm of the maxillary sinus. Squamous cell carcinoma (SCC) represents about $80-90 \%$ cases of the malignant tumours of the maxillary sinus or of the head and neck region.

\section{CONCLUSIONS}

Considering the preoperative clinical investigations, results and observation during surgery, a correct diagnosis should include a histopathological examination. The case report shows the necessity of CT or MRI scans, for complete correlation between the extension of the lesion, sinus cavity and oral cavity. Inner tumour disruption or pus can give a positive fine needle aspiration (FNAC) from tumour, which was positive in our patient. Complications may include perforation of the vestibular bone, nasal cavity and maxillary sinus ${ }^{5}$. A large cyst can cause complete destruction of the anterior palate and pyriform rim ${ }^{6}$.

Conflict of interest: The authors have no conflict of interest.

Contribution of authors: All authors have equally contributed to this work.

\section{REFERENCES}

1. Barnes L, Everson JW, Reichart P, Sidransky D. (eds.) World Health Organization. Classification of Tumors. Pathology and Genetics of Head and Neck Tumors. IARC Press: Lyon; 2005.

2. Kato H, Kanematsu M, Kusunoki Y, Shibata T, Murakami H, Mizuta K, et al. Nasoalveolar cyst: imaging findings in three cases. Clin Imaging. 2007;31(3):206-9.

3. Ely N, Sheehy EC, McDonald F. Nasopalatine duct cyst: a case report. Int J Paediatr Dent. 2001;11(2):135-7.

4. Hegde R, Shetty R. Nasopalatine duct cyst. J Indian Soc Pedod Prev Dent. 2006;24(Suppl 1):S31-2.

5. Scolozzi P, Martinez A, Richter M, Lombardi T. A nasopalatine duct cyst in a 7-year-old child. Pediatr Dent. 2008;30(6):530-4.

6. Sankar D, Muthusubramanian V, Nathan JA, Nutalapati RS, Jose YM, Kumar YN. Aggressive nasopalatine duct cyst with complete destruction of palatine bone. JPharm Bioallied Sci. 2016;8(Suppl 1):S185-S188. 